\title{
DEC2 expression antagonizes cisplatin-induced apoptosis in human esophageal squamous cell carcinoma
}

\author{
HIDENOBU SATO $^{1,2}$, YUNYAN WU ${ }^{1}$, YUKIO KATO ${ }^{3}$, QIANG LIU $^{1}$, HIDEAKI HIRAI ${ }^{1}$, \\ TADASHI YOSHIZAWA ${ }^{1}$, SATOKO MOROHASHI ${ }^{1}$, JUN WATANABE ${ }^{2}$ and HIROSHI KIJIMA ${ }^{1}$ \\ ${ }^{1}$ Department of Pathology and Bioscience, Hirosaki University Graduate School of Medicine; ${ }^{2}$ Department \\ of Pathologic Analysis, Division of Medical Life Sciences, Hirosaki University Graduate School of \\ Health Sciences, Hirosaki, Aomori 036-8562; ${ }^{3}$ Department of Dental and Medical Biochemistry, Institute \\ of Biomedical and Health Sciences, Hiroshima University, Hiroshima 734-8553, Japan
}

Received December 17, 2015; Accepted December 19, 2016

DOI: $10.3892 / \mathrm{mmr} .2017 .6571$

\begin{abstract}
Differentiated embryonic chondrocyte expressed gene 1 (DEC1) and differentiated embryonic chondrocyte expressed gene 2 (DEC2) belong to the Hairy/Enhancer of Split subfamily of basic helix-loop-helix factors. Previous studies have demonstrated that DEC proteins are involved in the regulation of circadian rhythms, response to hypoxia, and tumorigenesis. However, the roles of DEC1 and DEC2 in apoptosis of esophageal carcinoma remain unclear. In the present study, alterations in expression of apoptosis-related markers in human esophageal squamous cell carcinoma TE-11 cells treated with cisplatin were examined by western blot, while overall cell viability and apoptosis were analyzed by MTS assay and hematoxylin and eosin staining, respectively. Following cisplatin treatment, expression of DEC2 was downregulated, whereas expression of DEC1 was upregulated. DEC2 overexpression during cisplatin treatment markedly inhibited expression of the pro-apoptotic factor Bim and slightly increased the anti-apoptotic factor Bcl-xL. However, overexpression of DEC1 during cisplatin treatment failed to affect expression of these markers. Additionally, overexpression of DEC2 improved cell viability and decreased cell apoptosis induced by cisplatin. These results suggested that DEC2 exhibits anti-apoptotic effects in TE-11 esophageal squamous cell carcinoma cells. Inhibiting DEC2 may therefore have therapeutic potential for the treatment of esophageal cancer, in combination with cisplatin.
\end{abstract}

Correspondence to: Dr Yunyan Wu, Department of Pathology and Bioscience, Hirosaki University Graduate School of Medicine, 5 Zaifu-cho, Hirosaki, Aomori 036-8562, Japan

E-mail: yunyanwu@hirosaki-u.ac.jp

Key words: differentiated embryonic chondrocyte expressed gene 1, differentiated embryonic chondrocyte expressed gene 2, apoptosis, esophageal squamous cell carcinoma, TE-11 cells

\section{Introduction}

Global cancer statistics suggest that $\sim 455,800$ new cases of esophageal cancer (EC) were diagnosed in 2012, and $\sim 400,200$ people succumbed to this disease worldwide (1). EC is 3-4 times more common in males than females. EC is more malignant than other gastrointestinal cancers and is characterized by a variable geographic distribution of incidence and histological subtypes, including esophageal squamous cell carcinoma (ESCC) and esophageal adenocarcinoma (EAC) (1). ESCC is the most common subtype and accounts for over $90 \%$ of all EC cases in Asian countries, including China and Japan (2).

Cisplatin, a widely used platinum-containing chemotherapeutic drug, has been employed in the treatment of various types of cancer, and its primary function is induction of cell death by apoptosis (3). It is hypothesized that three different stages are involved in the apoptosis process: The initiation phase, the effector phase and the execution phase (4). The Bcl-2 protein family is a key regulator of the execution phase. There are $>30$ members of the Bcl-2 family of proteins reported in the literature, that are divided into the Bcl-2-like proteins, the Bcl2-associated X (Bax)-like proteins, and the Bcl-2 homology domain (BH3)-only proteins, according to their structure and function (5). When exposed to cytotoxic stimuli, BH3-only proteins initiate apoptosis by binding and inhibiting the anti-apoptotic Bcl-2-like proteins. This leads to the pro-apoptotic Bax-like proteins forming oligomers that permeabilize the mitochondrial outer membrane, resulting in the release of apoptogenic factors and activating the effector caspases to cause apoptosis (6). Bcl-2-interacting mediator of cell death (Bim), one of the $\mathrm{BH} 3$-only protein members, has been confirmed to have at least 6 isoforms (7). Among the various isoforms, $\mathrm{Bim}_{\mathrm{EL}}$ (extra long form), $\mathrm{Bim}_{\mathrm{L}}$ (long form) and $\mathrm{Bim}_{\mathrm{S}}$ (short form) are the most well-characterized $(5,8)$. Although all three isoforms promote apoptosis, they differ from each other in cytotoxicity and tissue expression distribution; $\mathrm{Bim}_{\mathrm{S}}$ is the most cytotoxic but not universally expressed, while $\mathrm{Bim}_{\mathrm{EL}}$ and $\mathrm{Bim}_{\mathrm{L}}$ are expressed in various tissues and cell types (9).

Basic helix-loop-helix transcription factors differentiated embryonic chondrocyte expressed gene 1 [DEC1; 
officially known as basic helix-loop-helix family member e40 (BHLHE40)] and differentiated embryonic chondrocyte expressed gene 2 [DEC2; officially known as basic helix-loop-helix family member e41 (BHLHE41)] are regulators of circadian rhythms, response to hypoxia, mediators of epithelial-mesenchymal transition, and apoptosis (10-13). The function of DEC1 in apoptosis is controversial, however previous studies have revealed that DEC2 functions as an anti-apoptotic factor in several cancer cells, such as breast cancer MCF-7 cells and oral cancer HSC-3 cells (13-15). In the present study, the role of DEC1 and DEC2 in cisplatin-induced apoptosis was assessed in human esophageal TE-11 cancer cells.

\section{Materials and methods}

Cell culture and treatment. The human ESCC cell line TE-11 was purchased from the RIKEN BioResource Center (Tsukuba, Japan) through the National Bio-Resource Project of the Ministry of Education, Culture, Sports, Science and Technology, Japan. The cells were cultured in RPMI Medium 1640 (Gibco; Thermo Fisher Scientific, Inc., Waltham, MA, USA) supplemented with $10 \%$ fetal bovine serum (Gibco; Thermo Fisher Scientific, Inc.) at $37^{\circ} \mathrm{C}$ in a humidified atmosphere of $95 \%$ air and $5 \% \mathrm{CO}_{2}$. Where noted, the cells were incubated with cisplatin (Sigma-Aldrich; Merck Millipore, Darmstadt, Germany) at various concentrations for $24 \mathrm{~h}$.

DEC1 and DEC2 overexpression. The expression plasmids for human DEC1 and DEC2 were donated by Dr Katusmi Fujimoto (Hiroshima University, Japan) (11). TE-11 cells were seeded at $5 \times 10^{4}$ cells per $35 \mathrm{~mm}$ well. DEC1 or DEC2 plasmid was transfected into the cells using Lipofectamine ${ }^{\circledR}$ LTX (Invitrogen; Thermo Fisher Scientific, Inc.) according to the manufacturer's instructions. Following transfection, the cells were incubated for $24 \mathrm{~h}$ and then treated with cisplatin at 20 or $40 \mu \mathrm{M}$ for an additional $24 \mathrm{~h}$. The cells were then used in the western blot analysis or the MTS assay.

Antibodies. Primary antibodies to DEC1 (cat. no. NB100-1800; Novus Biologicals, Ltd. Littleton, CO, USA; 1:5,000 dilution), DEC2 (H-72; cat. no. sc-32853; Santa Cruz Biotechnology, Inc., Dallas, TX, USA; 1:10,000 dilution), Bax (N-20; cat. no. sc-493; Santa Cruz Biotechnology, Inc.) and $\beta$-actin (cat. no. A5060; Sigma-Aldrich; Merck Millipore; 1:10,000 dilution) were used. Primary antibodies to poly ADP-ribose polymerase (PARP; cat. no. 9542; 1:5,000 dilution), cleaved caspase-3 (Asp175; cat. no. 9961; 1:1,000 dilution), cleaved caspase-8 (Asp391; cat. no. 9496; 1:1,5000 dilution), Bim (cat. no. 2819; 1:2,000 dilution), Bcl-2 (50E3; cat. no. 2870; 1:10,000 dilution) and Bcl-2-like 1 protein extra large (Bcl-xL; 54H6; cat. no. 2764; 1:10,000 dilution) were purchased from Cell Signaling Technology, Inc. (Danvers, MA, USA). Anti-rabbit IgG (cat. no. 17502) and anti-mouse IgG (cat. no. 17601) secondary antibodies were purchased from Immuno-Biological Laboratories Ltd. (Fujioka, Japan). Can Get Signal Immunoreaction Enhancer Solution (Toyobo Co., Ltd. Osaka, Japan) or Immunoshot Immunoreaction Enhancer Solution (Cosmobio Co., Ltd., Tokyo, Japan) were used to dilute the primary antibodies.
Western blot analysis. M-PER lysis buffer (Thermo Fisher Scientific, Inc.) was added to the cells cultured in a 6-well plate, and cells were incubated for $5 \mathrm{~min}$ at room temperature, with gentle agitation. The lysate was collected and transferred to a microcentrifuge tube and centrifuged at $12,000 \times \mathrm{g}$ for $10 \mathrm{~min}$ at $4^{\circ} \mathrm{C}$. The supernatant was collected and transferred to a new tube for analysis. Protein concentration was determined using the bicinchoninic acid (BCA) assay. The purified protein (10 $\mu \mathrm{g}$ per lane) were subjected to $10 \%$ SDS-PAGE, and the proteins were transferred onto polyvinylidene difluoride membranes (Immobilon P; Merck Millipore), which were then probed with primary antibodies at $4^{\circ} \mathrm{C}$ overnight. The membranes were subsequently washed with TBS containing Tween 20, and were incubated with secondary antibodies for $1 \mathrm{~h}$ at room temperature with agitation. Proteins of interest were visualized with enhanced chemiluminescence (ECL) reagents using the ECL, ECL-Prime, or ECL-Select Western Blotting Detection system (Amersham; GE Healthcare Life Sciences, Chalfont, UK). Densitometry was performed using ImageJ version 1.48 (National Institutes of Health, Bethesda, MD, USA). Each experiment was repeated 3 times.

Cell viability assay. TE-11 cells were seeded at a density of $2.5 \times 10^{3}$ into 96 -well plates. The cells were transfected with an empty plasmid (pcDNA) or the expression plasmids for DEC1 or DEC2 (DEC1 pcDNA or DEC2 pcDNA, respectively). Following $18 \mathrm{~h}$ of transfection, the cells were cultured with or without $40 \mu \mathrm{M}$ cisplatin for another $24 \mathrm{~h}$. Cell viability was assessed with the MTS assay, as previously described (16).

Hematoxylin and eosin $(H \& E)$ staining. Apoptosis was evaluated by $\mathrm{H} \& \mathrm{E}$ staining. Briefly, TE-11 cells at $70 \%$ confluency were transfected with DEC2 plasmid DNA for $18 \mathrm{~h}$, followed by treatment with $40 \mu \mathrm{M}$ of cisplatin for $24 \mathrm{~h}$. The cells were then fixed with $4 \%$ paraformaldehyde (Wako Pure Chemical Industries, Ltd., Osaka, Japan) in phosphate-buffered saline (PBS) for 15 min, permeabilized with $0.25 \%$ Triton $\mathrm{X}-100$ (Sigma-Aldrich; Merck Millipore) in PBS for $20 \mathrm{~min}$ and finally stained by $\mathrm{H} \& \mathrm{E}$.

Statistical analysis. Each experiment was repeated a minimum of three times. GraphPad Prism software version 7.02 (GraphPad Software, Inc., La Jolla, CA, USA) was used to perform one-way or two-way analyses of variance, followed by Dunnett's or Šidák's tests. Data are presented as the mean \pm standard deviation. $\mathrm{P}<0.05$ was considered to indicate a statistically significant difference.

\section{Results}

Effects of cisplatin on the expression of DEC1 and DEC2 in TE-11 cells. Cisplatin treatment resulted in different outcomes on the endogenous expression of DEC1 and DEC2 (Fig. 1A). Expression of DEC2 was decreased with 20 and $50 \mu \mathrm{M}$ cisplatin, whereas expression of DEC1 was increased in the same conditions (Fig. 1A). Treatment with $10 \mu \mathrm{M}$ cisplatin induced expression of cleaved PARP, cleaved caspase-8, $\operatorname{Bim}_{\mathrm{EL}}, \operatorname{Bim}_{\mathrm{L}}$ and $\mathrm{Bim}_{\mathrm{S}}$ (Fig. 1A). Treatment with 20 and $50 \mu \mathrm{M}$ cisplatin further increased the amounts of cleaved PARP, cleaved caspase-8, cleaved caspase-3, Bim and Bax, whereas 


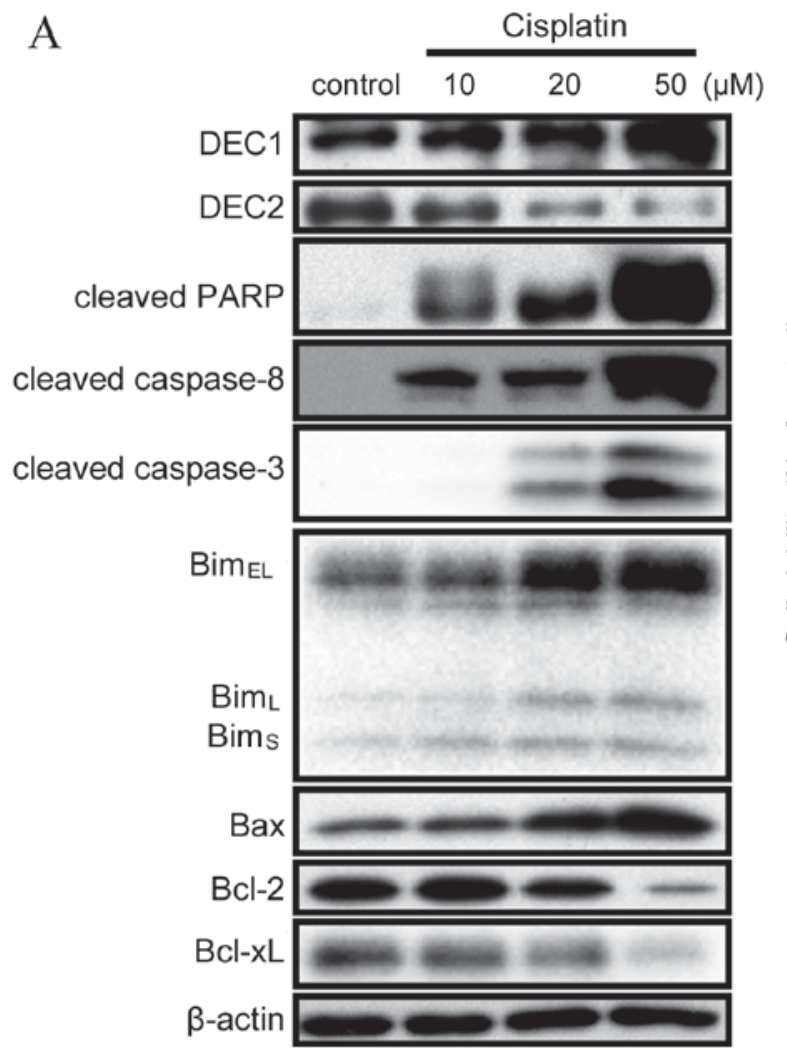

B

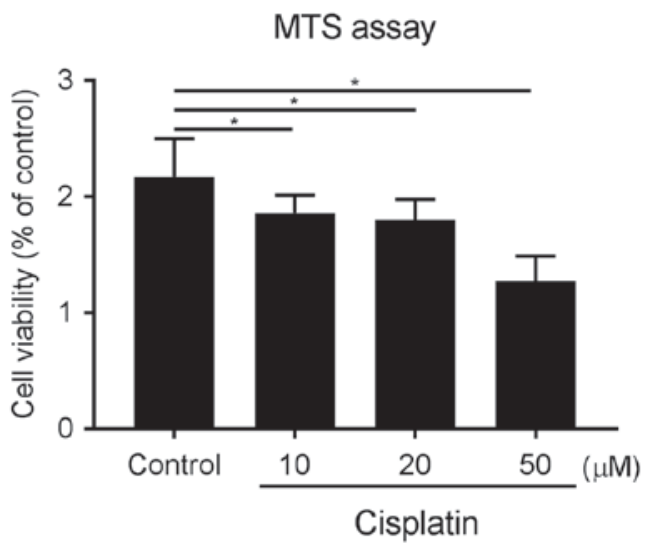

Figure 1. Cisplatin treatment affects expression of DEC1, DEC2 and apoptotic markers in TE-11 cells. (A) TE-11 cells were treated with 10, 20 and $50 \mu \mathrm{M}$ cisplatin for $24 \mathrm{~h}$. Cell lysates were subjected to western blot analysis for protein expression of DEC1, DEC2, cleaved PARP, cleaved caspase-8, cleaved caspase-3, Bim isoforms, Bax, Bcl-2, Bcl-xL and $\beta$-actin. (B) TE-11 cells were treated with 10, 20 and $50 \mu \mathrm{M}$ cisplatin for 24 h, and then cell viability was measured using the MTS-assay. Data are presented as the mean \pm standard deviation, from three independent experiments ( $\left.{ }^{*} \mathrm{P}<0.05\right)$. DEC, differentiated embryonic chondrocyte expressed gene; PARP, poly ADP-ribose polymerase; Bim, Bcl-2-interacting mediator of cell death; EL, extra long; L, long; S, short; $\mathrm{Bax}, \mathrm{Bcl}-2$ associated protein $\mathrm{X}$ apoptosis regulator; Bcl-2, B-cell lymphoma 2 apoptosis regulator; BcL-xL, Bcl-2-like 1 protein extra large.

it decreased the expression of Bcl-2 and Bcl-xL (Fig. 1A). In addition, the ratio of $\mathrm{Bax} / \mathrm{Bcl}-2$ protein expression was strongly increased with $50 \mu \mathrm{M}$ cisplatin (Fig. 1A). Treatment of TE-11 cells with 10, 20 and $50 \mu \mathrm{M}$ cisplatin was demonstrated to significantly reduce cell viability (Fig. 1B).

DEC2 inhibits Bim expression and apoptosis induced by cisplatin in TE-11 cells. The effect of DEC1 or DEC2 overexpression on apoptosis was examined by transient transfection of expression plasmids into TE-11 cells. DEC2 overexpression in the presence of $20 \mu \mathrm{M}$ cisplatin visibly decreased the amounts of cleaved PARP, cleaved caspase-3, cleaved caspase- 8 and $\operatorname{Bim}\left({ }_{E L},{ }_{L}\right.$, and $\left.{ }_{S}\right)$ in TE-11 cells, compared with cells transfected with empty vector control (Fig. 2A). Conversely, DEC2 overexpression slightly increased the expression of Bcl-xL compared with control, but had little effect on the expression of Bcl-2 (Fig. 2A). Cisplatin augmented DEC1 expression in a dose dependent manner (Fig. 1A); however, DEC1 overexpression had no significant effect on cleaved PARP (1.24-fold those of pcDNA-trasnsfected group) or cleaved caspase-8 (1.28-fold those of pcDNA-transfected group) protein expression levels under cisplatin treatment in TE-11 cells (Fig. 2B).

DEC2 overexpression inhibits cisplatin-induced cell death in TE-11 cells. Overexpression of DEC1 or DEC2 and cisplatin treatment were combined in TE-11 cells to investigate whether DEC proteins affect cell viability (Fig. 3). DEC2 overexpression significantly increased the number of live cells in the absence or presence of cisplatin compared with pcDNA control (Fig. 3A), whereas DEC1 overexpression had little effect (Fig. 3B). Finally, the apoptotic changes in TE-11 cells were examined by H\&E staining (Fig. 4). Cell mitoses were observed in the pcDNA- or DEC2 pcDNA-transfected cells without cisplatin treatment (Fig. 4, arrowheads), however, cisplatin significantly increased the number of apoptotic TE-11 cells (Fig. 4, arrows) and decreased the number of cell mitoses.

\section{Discussion}

In the present study, the roles of DEC1 and DEC 2 in the process of cisplatin-induced apoptosis of human esophageal carcinoma cells were analyzed. Cisplatin treatment increased expression of DEC1, but decreased expression of DEC2 in TE-11 cells. DEC2 overexpression in the presence of cisplatin markedly inhibited multiple apoptotic markers, including all three splice variants of $\mathrm{Bim}, \mathrm{Bim}_{\mathrm{EL}}, \mathrm{Bim}_{\mathrm{L}}$ and $\mathrm{Bim}_{\mathrm{S}}$, as well as cleaved caspase-3, cleaved caspase-8, and cleaved PARP. DEC2 had been demonstrated to inhibit apoptosis in the breast adenocarcinoma cell line MCF-7 (13) and the squamous cell carcinoma cell line HSC-3 (14). However, in another squamous cell carcinoma cell line, CA9-22, which demonstrates high endogenous expression of epidermal growth factor receptor (EGFR), DEC2 failed to affect cisplatin-induced apoptosis (14). In order to address this discrepancy, EGFR expression was analyzed 

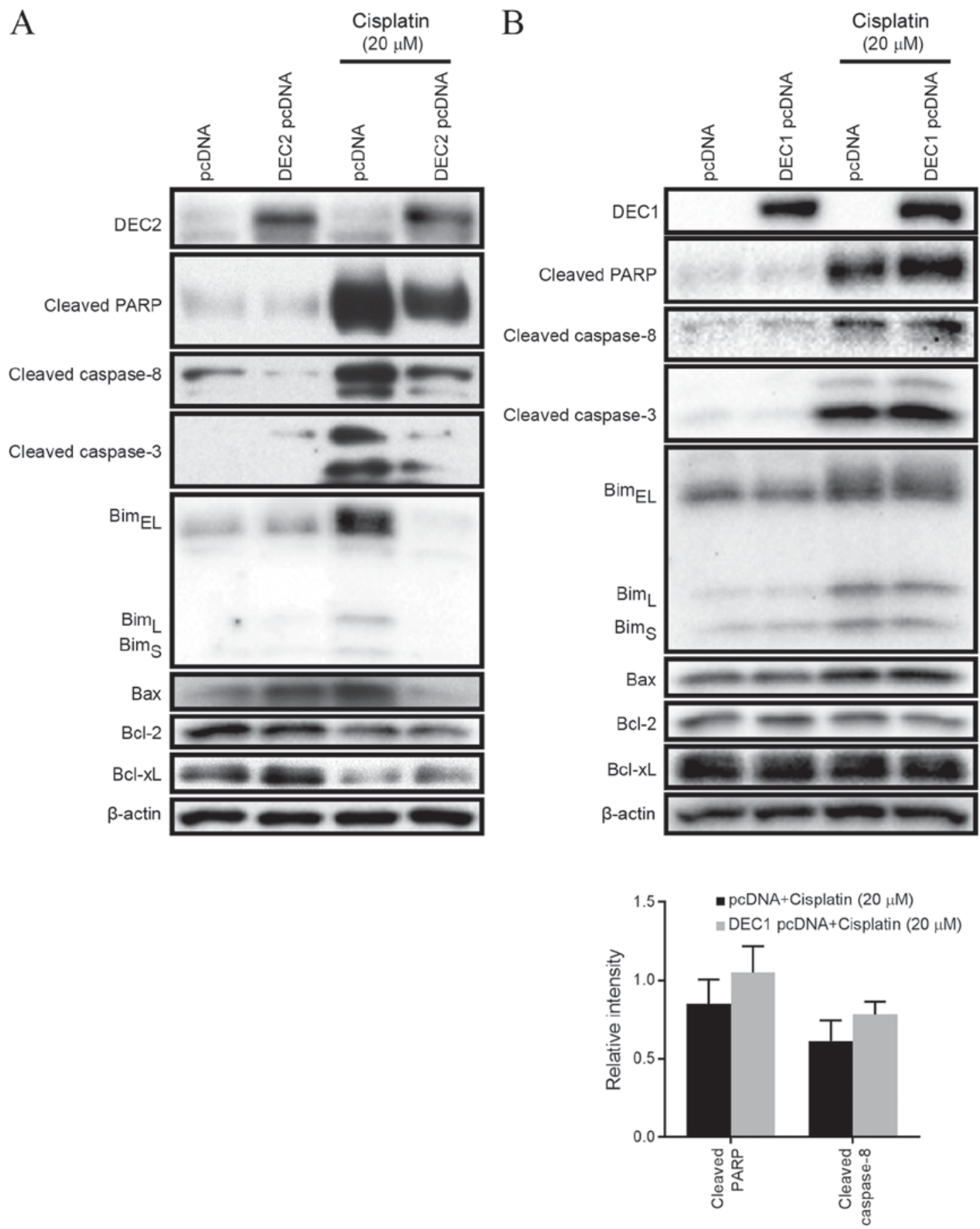

Figure 2. DEC2 inhibits expression of apoptotic markers following cisplatin treatment. TE-11 cells were transfected for $24 \mathrm{~h}$ with pcDNA, (A) DEC2 pcDNA or (B) DEC1 pcDNA. Transfected cells were incubated with $20 \mu \mathrm{M}$ cisplatin for an additional $24 \mathrm{~h}$, and then lysed. Cell lysates were subjected to western blot analysis for protein expression of DEC1, DEC2, cleaved PARP, cleaved caspase- 8 , cleaved caspase-3, Bim isoforms, Bax, Bcl-2, Bcl-xL and $\beta$-actin. Data are presented as the mean \pm standard deviation, from three independent experiments. DEC, differentiated embryonic chondrocyte expressed gene; pcDNA, empty vector control; DEC2 pcDNA, DEC2 overexpression vector; DEC1 pcDNA, DEC1 overexpression vector; PARP, poly ADP-ribose polymerase; Bim, Bcl-2-interacting mediator of cell death; EL, extra long; L, long; S, short; Bax, Bcl-2 associated protein X apoptosis regulator; Bcl-2, B-cell lymphoma 2 apoptosis regulator; BcL-xL, Bcl-2-like 1 protein extra large.

in TE-11 cells. A431 cells, which are known to express high levels of EGFR, were used as a positive control. TE-11 cells exhibited significantly lower amount of EGFR expression than A431 cells (data not shown), which might explain why DEC2 functions as an inhibitor of apoptosis in TE-11 cells, but not in the previously reported CA9-22 cells.

DEC protein expression is modulated in normal and cancerous cells by several types of stimulation, such as cytokines (15), anticancer reagents (13), and hypoxia (12). DEC2 has also been identified as one of the members of the circadian genes, which exhibit rhythmic expression during the day, not only in normal cells but also in cancer cells $(11,17)$. As a result, scientists and clinicians are beginning to take chronochemotherapy into consideration, which is a time-dependent manner of treatment administration to cancer patients. Cisplatin is one of the most commonly used chemotherapeutic 

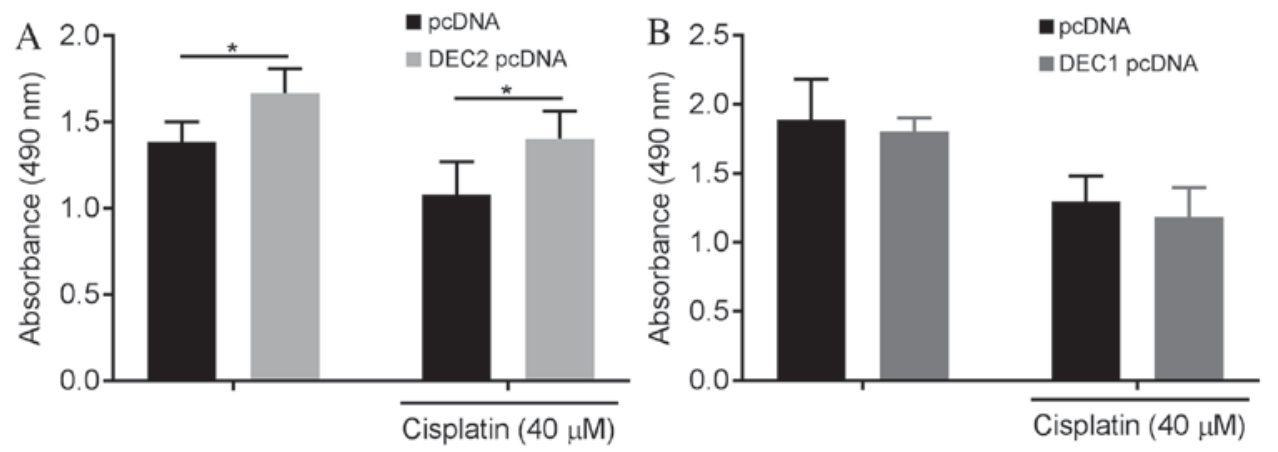

Figure 3. DEC2 overexpression inhibits cell death induced by cisplatin. TE-11 cells were transfected for $24 \mathrm{~h}$ with pcDNA, (A) DEC2 pcDNA or (B) DEC1 pcDNA. Transfected cells were treated with $40 \mu \mathrm{M}$ cisplatin for an additional $24 \mathrm{~h}$, and then cell viability was measured using the MTS-assay. Data are presented as the mean \pm standard deviation, from three independent experiments $($ "P $<0.05)$. DEC, differentiated embryonic chondrocyte expressed gene; pcDNA, empty vector control; DEC2 pcDNA, DEC2 overexpression vector; DEC1 pcDNA, DEC1 overexpression vector.

$(-)$

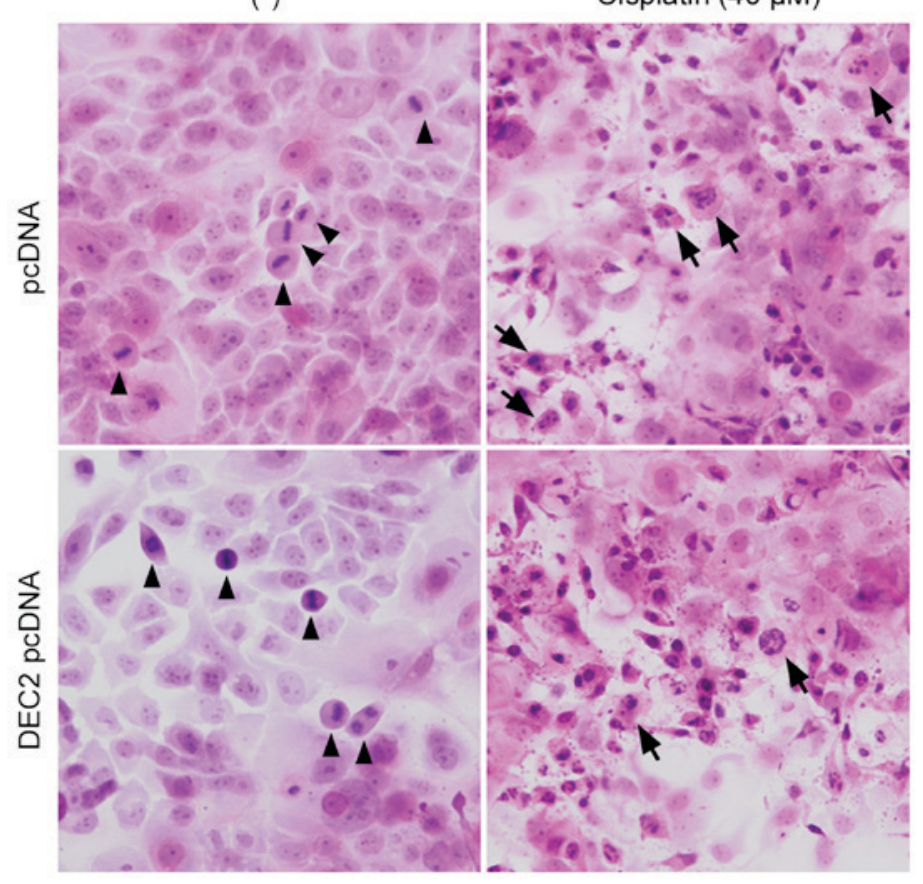

Figure 4. DEC2 overexpression inhibits apoptosis. Apoptosis was visualized by hematoxylin and eosin staining in TE-11 cells transfected with either pcDNA or DEC2 pcDNA, following treatment with either DMSO or $40 \mu \mathrm{M}$ cisplatin for $24 \mathrm{~h}$. Arrow heads indicate mitotic cells and arrows indicate apoptotic cells. DEC, differentiated embryonic chondrocyte expressed gene; pcDNA, empty vector control; DEC2 pcDNA, DEC2 overexpression vector.

drugs for the treatment of a variety of tumors. However, side effects including tumor resistance and nephrotoxicity greatly limit its use. It has been reported that cisplatin transporter molecules exhibit varying activity levels over a 24 -h period, with greater expression in the evening compared with the morning (18). Future studies are required into the association between DEC2 and these transporter molecules, and the functions and underlying mechanisms of DEC2 in regulating apoptosis, to evaluate its full potential as a target in esophageal carcinoma chemotherapy.

\section{Acknowledgements}

This work was supported by JSPS KAKENHI, Grants-in-Aid for Young Scientists from the Ministry of Education, Culture, Sports, Science, and Technology of Japan (grant no. 26870028) and a Grant for Hirosaki University Institutional Research (grant no. 5310139303).

\section{References}

1. Torre LA, Bray F, Siegel RL, Ferlay J, Lortet-Tieulent J and Jemal A: Global cancer statistics, 2012. CA Cancer J Clin 65: 87-108, 2015.

2. Lin Y, Totsuka Y, He Y, Kikuchi S, Qiao Y, Ueda J, Wei W, Inoue $\mathrm{M}$ and Tanaka $\mathrm{H}$ : Epidemiology of esophageal cancer in Japan and China. J Epidemiol 23: 233-242, 2013.

3. Dasari S and Tchounwou PB: Cisplatin in cancer therapy: Molecular mechanisms of action. Eur J Pharmacol 740: 364-378, 2014.

4. Eastman A: The Mechanism of Action of Cisplatin: From Adducts to Apoptosis. In: Cisplatin Verlag Helvetica Chimica Acta, pp111-134, 2006.

5. Cory S and Adams JM: The Bcl2 family: Regulators of the cellular life-or-death switch. Nat Rev Cancer 2: 647-656, 2002. 
6. Czabotar PE, Lessene G, Strasser A and Adams JM: Control of apoptosis by the BCL-2 protein family: Implications for physiology and therapy. Nat Rev Mol Cell Biol 15: 49-63, 2014.

7. Marani M, Tenev T, Hancock D, Downward J and Lemoine NR: Identification of novel isoforms of the bh3 domain protein bim which directly activate bax to trigger apoptosis. Mol Cell Biol 22: 3577-3589, 2002.

8. O'Connor L, Strasser A, O'Reilly LA, Hausmann G, Adams JM, Cory S and Huang DC: Bim: A novel member of the Bcl-2 family that promotes apoptosis. EMBO J 17: 384-395, 1998.

9. O'Reilly LA, Cullen L, Visvader J, Lindeman GJ, Print C, Bath ML, Huang DC and Strasser A: The Proapoptotic BH3-only protein bim is expressed in hematopoietic, epithelial, neuronal, and germ cells. Am J Pathol 157: 449-461, 2000.

10. Wu Y, Sato F, Yamada T, Bhawal UK, Kawamoto T, Fujimoto K, Noshiro M, Seino H, Morohashi S, Hakamada K, et al: The BHLH transcription factor DEC1 plays an important role in the epithelial-mesenchymal transition of pancreatic cancer. Int $\mathbf{J}$ Oncol 41: 1337-1346, 2012.

11. Honma S, Kawamoto T, Takagi Y, Fujimoto K, Sato F, Noshiro M, Kato Y and Honma K: Dec1 and Dec2 are regulators of the mammalian molecular clock. Nature 419: 841-844, 2002.

12. Miyazaki K, Kawamoto T, Tanimoto K, Nishiyama M, Honda H and Kato Y: Identification of functional hypoxia response elements in the promoter region of the DEC1 and DEC2 genes. J Biol Chem 277: 47014-47021, 2002.

13. Wu Y, Sato F, Bhawal UK, Kawamoto T, Fujimoto K, Noshiro M, Morohashi S, Kato Y and Kijima H: Basic helix-loop-helix transcription factors DEC1 and DEC2 regulate the paclitaxel-induced apoptotic pathway of MCF-7 human breast cancer cells. Int J Mol Med 27: 491-495, 2011.
14. Wu Y, Sato F, Bhawal UK, Kawamoto T, Fujimoto K, Noshiro M, Seino H, Morohashi S, Kato Y and Kijima H: BHLH transcription factor DEC2 regulates pro-apoptotic factor Bim in human oral cancer HSC-3 cells. Biomed Res 33: 75-82, 2011.

15. Liu Y, Sato F, Kawamoto T, Fujimoto K, Morohashi S, Akasaka H, Kondo J, Wu Y, Noshiro M, Kato Y and Kijima H: Anti-apoptotic effect of the basic helix-loop-helix (bHLH) transcription factor DEC2 in human breast cancer cells. Genes Cells 15: 315-325, 2010.

16. Wu Y, Sato H, Suzuki T, Yoshizawa T, Morohashi S, Seino H, Kawamoto T, Fujimoto K, Kato Y and Kijima H: Involvement of c-Myc in the proliferation of MCF-7 human breast cancer cells induced by bHLH transcription factor DEC2. Int J Mol Med 35: 815-820, 2015.

17. Sato F, Bhawal UK, Kawamoto T, Fujimoto K, Imaizumi T, Imanaka T, Kondo J, Koyanagi S, Noshiro M, Yoshida H, et al: Basic-helix-loop-helix (bHLH) transcription factor DEC2 negatively regulates vascular endothelial growth factor expression. Genes Cells 13: 131-144, 2008.

18. Sorensen DN, Dakup PP and Gaddameedhi S: Chronopharmacology of cisplatin: Role of the circadian rhythm in modulating the cisplatin transporters levels. The FASEB Journal 30 (1 Suppl): S935, 2016 